\title{
Quality Indicators for the Detection of Helicobacter pylori-Negative Early Gastric Cancer: A Retrospective Observational Study
}

\author{
Fumiaki Ishibashi ${ }^{1,2}$, Konomi Kobayashi ${ }^{1,3}$, Keita Fukushima ${ }^{2,3}$, Ryu Tanaka ${ }^{2}$, Tomohiro Kawakami ${ }^{1}$, Junko Kato ${ }^{2}$ and \\ Kazuaki Sugihara ${ }^{2}$ \\ ${ }^{1}$ Koganei Tsurukame Clinic, Endoscopy Center, Tokyo, ${ }^{2}$ Shinjuku Tsurukame Clinic, Digestive Disease Center, Tokyo, ${ }^{3}$ Mirraza Shinjuku \\ Tsurukame Clinic, Tokyo, Japan
}

Background/Aims: While Helicobacter pylori (HP)-negative gastric cancer is frequently reported, little is known about the predictors for detecting HP-negative early gastric cancer (EGC). We aimed to evaluate the predictors for the detection of HP-negative EGC. Methods: We retrospectively reviewed 13,477 consecutive asymptomatic cases where upper endoscopy was performed by nine physicians from April 2017 to March 2019 and analyzed the detection rate of high-risk lesions (HRLs), including EGC, tubular adenoma, and lymphoma, according to the status of HP infection. The observation time was corrected for multiple regression analyses. Results: For all physicians, the average observation time for screening HP-eradicated and -naïve patients was shorter than that for screening HP-positive patients $(p<0.05)$. Multiple regression analyses revealed that the observation time in the three groups was an independent predictor for detecting HRLs in HP-eradicated patients $(p=0.03106,0.01263$, and 0.02485 , respectively), while experience of endoscopy was an independent predictor for detecting HRLs in HP-naïve patients ( $p=0.02638$ ).

Conclusions: While observation time during screening endoscopy was a quality indicator for detecting HRLs in HP-eradicated patients, experience of endoscopy was a quality indicator for detecting HRLs in HP-naïve patients. Clin Endosc 2020;53:698-704

Key Words: Detection rate; Early gastric cancer; Helicobacter pylori; Quality indicator

\section{INTRODUCTION}

Helicobacter pylori (HP) is a major cause of carcinogenesis in the gastric mucosa, and the majority of intestinal-type early gastric cancers (I-EGCs) develop in the HP-positive gastric mucosa. ${ }^{1-3}$ Moreover, diffuse-type EGCs (D-EGCs) can be detected in the HP-positive gastric mucosa. While current HP infection is a risk factor for both I-EGC and D-EGC, successful therapy and HP eradication decreases the prevalence. ${ }^{4 \cdot 6}$

Received: October 21, 2019 Revised: November 13, 2019

Accepted: December 10, 2019

Correspondence: Fumiaki Ishibashi

Koganei Tsurukame Clinic, Endoscopy Center, 6-14-28-3F, Honcho, Koganei-shi, Tokyo, 184-0004, Japan

Tel: +81-42-386-3757, Fax: +81-42-386-7390, E-mail: ishibashi@tsurukamekai.jp ORCID: https://orcid.org/0000-0003-4065-7958

cc This is an Open Access article distributed under the terms of the Creative Commons Attribution Non-Commercial License (http://creativecommons.org/ licenses/by-nc/3.0) which permits unrestricted non-commercial use, distribution, and reproduction in any medium, provided the original work is properly cited.
Since eradication therapy of HP has been performed and public hygiene has improved worldwide, the proportion of people carrying HP has gradually decreased, and subsequently the likelihood for encountering HP-negative EGCs, including HPnaïve EGCs, has increased. ${ }^{7,8}$ Close follow-up after successful HP-eradication therapy revealed the possibility that EGCs could be detected in uninflamed gastric mucosa. ${ }^{9}$ Additionally, various types of HP-naïve gastric cancers, including gastric adenocarcinoma of the fundic gland type (GA-FG) and pure signet ring cell carcinoma, have been reported. ${ }^{710-12}$ However, it is fairly difficult to detect HP-negative gastric cancers, including HP-naïve EGCs, compared to HP-associated EGCs because the characteristics of such cancer are unclear and atypical. ${ }^{713}$ In addition, physicians tend to pay less attention to lesions during screening HP-naïve gastric mucosa.

In Japan, gastric cancer screening has been widely performed. Recently, it was suggested that screening by endoscopy is more effective than the barium swallow test with respect to detecting gastric cancer and reducing mortality rates due 
to gastric cancers. ${ }^{14-16}$ Based on this evidence, screening by endoscopy has been willingly chosen for the municipality based civilian medical checkup. In addition, a number of private companies promote the medical checkup of their employees, including an examination by upper endoscopy. Consequently, endoscopy centers for gastric cancer screening are required to perform a number of endoscopic examinations a day in a relatively short time; thus, missing EGC generated in HP-eradicated or -naïve mucosa is considered likely. Moreover, the examination time of an upper endoscopy was reported to be a quality indicator for upper endoscopy. ${ }^{17-19}$ However, it is unknown whether the shortness of the examination time influences the detection rate of EGCs, especially in the HP-negative stomach. Furthermore, the detection rate of EGCs depends on the frequency of biopsy and the physician's experience of endoscopy. ${ }^{20}$ In this study, we aimed to find quality indicators for detecting EGCs generated in the HP-negative stomach.

\section{MATERIALS AND METHODS}

\section{Subjects}

A total of 13,536 cases where upper endoscopy was performed from April 2017 to March 2019 were found in the endoscopic registry of Koganei Tsurukame Clinic; the data for these cases were retrospectively reviewed. All examinations were performed as part of the medical checkup and the subjects did not show any symptoms. In total, 35 patients who underwent surgical resection of the stomach and 24 patients who had food debris in their stomach were excluded. Conse- quently, 13,477 cases where upper endoscopy was performed were analyzed in this study.

\section{Endoscopic procedures}

All physicians were trained based on the program of the Japan Gastroenterological Endoscopy Society. The method of observation of the stomach was fixed in a unified manner: The mucus of the whole stomach was carefully removed with a cleaning solution, including mucolytic agents (Dimethicone), following which, each part of the stomach from the cardia to the pylorus was observed. All physicians were obliged to take at least 40 pictures of each location of the stomach. A specialist of endoscopy was defined as a board-certified member of the Japan Gastroenterological Endoscopy Society. The experience of endoscopy was defined as the elapsed year after starting training based on the Japan Gastroenterological Endoscopy Society.

\section{Data collection}

Detailed information of each case, including the physician who performed the examination, use of sedative agents (including propofol, midazolam, and pethidine hydrochloride), observation time, biopsy taken during the examination, and the extent of atrophic gastritis, was taken. The observation times of the stomach were retrospectively calculated as the difference between the time that the first and last pictures were taken. If a biopsy was taken, this time during the procedure was excluded. The results of the biopsy were referred to in corresponding patients' medical records. Detected lesions, including EGCs, neuroendocrine tumors, lymphomas, other

Table 1. Physician and Patient Characteristics

\begin{tabular}{|c|c|c|c|c|c|c|c|c|c|}
\hline \multirow[b]{2}{*}{ Physician } & \multicolumn{3}{|c|}{ Physician characteristics } & \multicolumn{6}{|c|}{ Patient characteristics } \\
\hline & Sex & $\begin{array}{c}\text { Experience } \\
\text { of endoscopy } \\
(y r)\end{array}$ & Certified & $\begin{array}{l}\text { Number of } \\
\text { case (total) }\end{array}$ & $\begin{array}{l}\text { Number } \\
\text { of cases } \\
\text { (per day) }\end{array}$ & Age & $\operatorname{Sex}(M: F)$ & $\begin{array}{c}\text { Sedative } \\
(\%)\end{array}$ & $\begin{array}{c}\text { Status of Helicobacter pylori } \\
\text { infection (positive/ } \\
\text { eradicated/naïve) (\%) }\end{array}$ \\
\hline A & $\mathrm{M}$ & 8 & Yes & 2,387 & 25.8 & 48 & $1.28: 1$ & 62.3 & $71 / 402 / 1,914(3.0 / 16.8 / 80.2)$ \\
\hline B & M & 6 & No & 2,069 & 22.2 & 46.2 & $1.16: 1$ & 60.1 & $66 / 343 / 1,660(3.2 / 16.6 / 80.2)$ \\
\hline C & M & 12 & Yes & 611 & 23.2 & 47.4 & $1.3: 1$ & 67.3 & 21/91/499 (3.4/14.9/81.7) \\
\hline $\mathrm{D}$ & $\mathrm{F}$ & 9 & Yes & 2,049 & 20.8 & 47.1 & $1.22: 1$ & 59.9 & $73 / 263 / 1,712(3.6 / 12.8 / 83.6)$ \\
\hline $\mathrm{E}$ & $\mathrm{F}$ & 5 & No & 2,091 & 23.4 & 48.6 & $1.21: 1$ & 63.4 & $69 / 309 / 1,713(3.3 / 14.8 / 81.9)$ \\
\hline $\mathrm{F}$ & M & 8 & Yes & 635 & 26.6 & 46.3 & $1.24: 1$ & 62.2 & $20 / 136 / 479(3.2 / 21.4 / 75.4)$ \\
\hline G & $\mathrm{F}$ & 8 & Yes & 603 & 23.8 & 46.2 & $1.32: 1$ & 65.2 & $17 / 135 / 451(2.8 / 22.5 / 74.7)$ \\
\hline $\mathrm{H}$ & M & 4 & No & 681 & 24.8 & 47.8 & $1.36: 1$ & 64.8 & $20 / 151 / 510(3.0 / 22.1 / 74.9)$ \\
\hline I & M & 14 & Yes & 2,351 & 25.4 & 47.2 & $1.09: 1$ & 59.2 & $70 / 479 / 1,802(3.0 / 20.4 / 76.6)$ \\
\hline Total & & & & 13,477 & & & & & $427 / 2,309 / 10,740$ \\
\hline Average & & & & & 24 & 47.2 & 1.24: 1 & 62.7 & $(3.2 / 17.1 / 79.7)$ \\
\hline
\end{tabular}

Certified: board-certified member of Japan Gastroenterological Endoscopy Society; Sedative: ratio of using sedative agents. 
types of gastric cancer, and intestinal adenomas, were defined as high-risk lesions (HRLs).

\section{Status of HP infection}

The status of HP infection was tested by serum anti-HP IgG antibody level, fecal HP antigen, urea breath test, and direct observation of biopsy sample. A serum anti-HP IgG antibody level $<3 \mathrm{~mL} / \mathrm{IU}$ was regarded as negative for infection, whereas positive for HP infection was judged based on the positive results of at least two distinct tests. All patients were questioned for their past HP eradication therapy. In addition, the degree of atrophy of the stomach was verified in order to validate whether the individual's answer for their experience of HP eradication was consistent with the change in the gastric mucosa. The extent of atrophic gastritis was described according to the Kimura-Takemoto classification. ${ }^{21}$ The study protocol was approved by the Ethics Committee of the Tsurukame Clinic (Approval number: 1801).

\section{Statistical analyses}

All statistical analyses were performed with $\mathrm{R} 3.3 .3$. $^{22} \mathrm{~A}$ one-way ANOVA was performed to compare data averages between two independent groups, and the results were expressed in terms of mean \pm standard error. Regression analyses were performed for explanatory variables with significant differences, and p-values were evaluated. Pearson's correlation analyses were performed for two distinct objective variables, and the results were described as correlation coefficient ( $r$ ) and $p$-value. $P$-values less than 0.05 were regarded as statistically significant. A multiple linear regression model with two distinct explanatory variables was used to establish the predic- tion model of the detection rate of HRL.

\section{RESULTS}

\section{Physician and patient characteristics}

A total of 13,477 cases of upper endoscopy, including examinations for both outpatients and medical checkup, were performed by nine physicians. The characteristics of the patients, as well as the physicians who performed the endoscopies are shown in Table 1. The mean number of cases that physicians performed a day was 24.0. The patients' mean age was 47.2 years old, and the ratio of male to female was 1.24:1. On the basis of the existence of atrophic gastritis and examinations for determining HP infection, the status of HP infection was current among 427 cases (3.2\%), past infection among 2,309 cases (17.1\%), and uninfected among 10,740 cases $(79.7 \%)$. There were no significant differences in patient background among the examining physicians. Notably, there were no significant differences in baseline characteristics for each group; HP-positive, -eradicated, and -naïve.

\section{Observation time for stomach screening was shorter in the HP-eradicated and -naïve patients compared to the HP-positive patients}

To address whether the observation time varies according to the status of HP infection, we compared the observation time of the HP-positive and -negative patients for all physicians. We found that the observation time in the HP-eradicated and -naive patients was significantly shorter for all physicians ( $p<0.05$, one-way ANOVA) (Table 2 ).

Table 2. Examination Summary

\begin{tabular}{|c|c|c|c|c|c|c|c|c|c|}
\hline \multirow[b]{2}{*}{ Physician } & \multicolumn{4}{|c|}{ Time (sec) } & \multirow[b]{2}{*}{ Biopsy (\%) } & \multicolumn{4}{|c|}{ HRL } \\
\hline & Total & $\mathrm{HP}+$ & $\begin{array}{c}\text { HP- } \\
\text { eradicated }^{\mathrm{a})}\end{array}$ & HP-naïve ${ }^{\text {a) }}$ & & Total (\%) & $\mathrm{HP}+(\%)$ & $\begin{array}{c}\text { HP- } \\
\text { eradicated (\%) }\end{array}$ & $\begin{array}{c}\text { HP- } \\
\text { naïve (\%) }\end{array}$ \\
\hline A & 252.9 & 293.4 & 238.9 & 225.8 & 25.3 & $8(0.34)$ & $2(2.82)$ & $3(0.75)$ & $3(0.16)$ \\
\hline B & 236.7 & 275.2 & 234.5 & 221.2 & 15 & $7(0.34)$ & $3(4.55)$ & $3(0.87)$ & $1(0.06)$ \\
\hline $\mathrm{C}$ & 211.4 & 257.2 & 198 & 183.2 & 23.7 & $3(0.49)$ & $0(0)$ & $1(1.1)$ & $2(0.4)$ \\
\hline $\mathrm{D}$ & 209.9 & 253 & 198.6 & 185.4 & 7.1 & $7(0.34)$ & $2(2.74)$ & $3(1.14)$ & $2(0.12)$ \\
\hline E & 206.9 & 270 & 195.3 & 182.1 & 9.2 & $6(0.29)$ & $2(2.9)$ & $2(0.65)$ & $2(0.12)$ \\
\hline $\mathrm{F}$ & 204.1 & 257.8 & 184.2 & 170.3 & 25.3 & $1(0.16)$ & $0(0)$ & $1(0.74)$ & $0(0)$ \\
\hline G & 193.8 & 231.7 & 175.4 & 166.2 & 24.9 & $1(0.17)$ & $1(5.88)$ & $0(0)$ & $0(0)$ \\
\hline $\mathrm{H}$ & 191.3 & 245 & 173.6 & 164.5 & 30.1 & 0 & $0(0)$ & $0(0)$ & $0(0)$ \\
\hline I & 184.1 & 239.8 & 170.1 & 162.8 & 55.3 & $4(0.17)$ & $2(2.86)$ & $2(0.42)$ & $0(0)$ \\
\hline Total/Average & 210.1 & 257 & 196.5 & 184.6 & 24 & $37(0.27)$ & $12(2.41)$ & $15(0.63)$ & $10(0.095)$ \\
\hline
\end{tabular}

HP, Helicobacter pylori; HP+, Helicobacter pylori-positive; HRL, high-risk lesion.

${ }^{\text {a) }} \mathrm{P}<0.05$, compared to time of $\mathrm{HP}+$ (one-way ANOVA). 


\section{Observation time and experience of endoscopy cor- relate with the detection rate of high-risk lesions in HP-eradicated and -naïve patients}

We explored the relationship between various physician factors and the detection rate of HRLs found in our registry. Among the 13,477 cases of our registry, 23 cases of HRLs, including EGCs, were found (Table 2). The detection rate of HRLs in the HP-positive patients was $2.41 \%$ (12 cases; 3 cases of D-EGC, 6 cases of I-EGC, and 3 cases of intestinal adenoma). In contrast, the detection rate of HRLs in the HP-eradicated and -naive patients was much lower at $0.63 \%$ (14 cases; 3 cases of D-EGC, 7 cases of I-EGC, and 4 cases of intestinal adenoma) and $0.095 \%$, respectively (10 cases; 3 cases of GAFG, 1 case of raspberry like tumor, 1 case of neuroendocrine tumor, 2 cases of mucosa-associated lymphoid tissue lymphoma, 2 cases of D-EGC, and 1 case of gastric-type I-EGC) compared to HP-positive patients. There was no significant difference in the pathological characteristics of the cancers in the three groups. Correlation analysis revealed that experience of endoscopy correlated with the detection rate of HRLs in the HP-naive patients ( $r=0.7273$; Table 3). The observation time in the HP-positive, -eradicated, and -naïve patients only correlated with the detection rate of HP-eradicated HRLs $(\mathrm{r}=0.713,0.7827$, and 0.7323 , respectively). These results suggest that observation time and experience of endoscopy are quality indicators for the detection of HRLs in HP-eradicated and -naive patients.

\section{Observation time and experience of endoscopy are quality indicators for detecting high-risk lesions in HP-negative patients}

To confirm the possibility that observation time and experience of endoscopy are predictors for the detection rate of HRLs in HP-eradicated and -naïve patients, we employed regression analysis for each explanatory variable (Table 4). Single regression analysis revealed that observation time

Table 3. Results of Correlation Analysis between Explanatory Variables and the High-Risk Lesion Detection Rate

\begin{tabular}{lcccc}
\hline & \multicolumn{4}{c}{ Detection rate of HRL } \\
\cline { 2 - 5 } & Total & HP-positive & HP-eradicated & HP-naïve \\
\hline Sex & 0.04798 & 0.5648 & -0.06976 & -0.1026 \\
Experience of endoscopy & 0.6371 & -0.06509 & 0.5587 & 0.7273 \\
Certified & -0.05964 & 0.08052 & -0.05516 & -0.1207 \\
Use of sedative agents & 0.02868 & -0.1991 & -0.1385 & 0.2746 \\
Number of case (a day) & -0.5406 & -0.4135 & -0.4362 & -0.4651 \\
Observation time (HP-positive) & 0.6586 & 0.05001 & 0.713 & 0.5014 \\
Observation time (HP-eradicated) & 0.816 & 0.3137 & 0.7827 & 0.5965 \\
Observation time (HP-naive) & 0.7796 & 0.3476 & 0.7323 & 0.5561 \\
Frequency of biopsy & -0.2999 & -0.0918 & -0.3101 & -0.2971 \\
\hline
\end{tabular}

The number indicates the r-value.

HP, Helicobacter pylori; HRL, high-risk lesion.

Table 4. Results of Regression Analysis for Determining the Factors for Predicting the High-Risk Lesion Detection Rate

\begin{tabular}{lcccc}
\hline & \multicolumn{4}{c}{ Detection rate of HRL } \\
\cline { 2 - 5 } & Total & HP-positive & HP-eradicated & HP-naïve \\
\hline Experience of endoscopy & $0.7285 /-0.1219$ & $0.8678 /-0.138$ & $0.1179 / 0.2138$ & $0.02638 / 0.4617$ \\
Observation time (HP-positive) & $0.05372 / 0.3529$ & $0.8983 /-0.14$ & $0.03106 / 0.4382$ & $0.1691 / 0.1445$ \\
Observation time (HP-eradicated) & $0.007306 / 0.6182$ & $0.4111 /-0.3041$ & $0.01263 / 0.5574$ & $0.08995 / 0.2638$ \\
Observation time (HP-naïve) & $0.01324 / 0.5517$ & $0.3594 /-0.004792$ & $0.02485 / 0.4701$ & $0.12 / 0.2106$ \\
Experience + observation time (HP-positive) & $0.01762 / 0.653$ & $0.9769 /-0.323$ & $0.02057 / 0.6347$ & $0.02853 / 0.5926$ \\
Experience + observation time (HP-eradicated) & $0.0004782 / 0.8957$ & $0.01395 / 0.679$ & $0.008093 / 0.7323$ & $0.01395 / 0.679$ \\
Experience + observation time (HP-naive) & $0.000583 / 0.8886$ & $0.655 /-0.158$ & $0.01242 / 0.6913$ & $0.01396 / 0.6789$ \\
\hline
\end{tabular}

The number indicates $p$-value/adjusted r-squared.

HP, Helicobacter pylori; HRL, high-risk lesion. 
during screening HP-positive, -eradicated, and -naïve stomach was an independent predictors for detecting HRLs in the HP -eradicated patients ( $p=0.03106,0.01263$, and 0.02485 , respectively). In addition, experience of endoscopy was an independent predictor for detecting HRLs in the HP-naive patients ( $p=0.02638$ ). Subsequently, we employed the same dataset for the multiple regression analysis to estimate the most efficient combinations of explanatory variables. As a result, the combination of observation time and experience of endoscopy improved the prediction of HRLs in HP-naïve patients (Table 4). By using the equation on the basis of the result of Table 4 , we estimated the detection rate of HRLs according to experience of endoscopy and observation time in the HP-naïve stomach (Table 5). This result suggested that less-experienced physicians could improve their detection rate of HRLs by taking sufficient time for screening. Furthermore, we validated this established model through the observed-predicted plot, and subsequently proved that the actual detection rates of HP-negative HRLs by physicians were well predicted (Fig. 1).

\section{DISCUSSION}

In contrast to the knowledge of the quality indicators for colonoscopy, little is known about the quality indicators for detecting EGCs. ${ }^{23} \mathrm{~A}$ few studies have revealed that several factors, including observation time and frequency of biopsy, are quality indicators for detecting EGCs. In this study, we aimed to validate previously reported quality indicators for detecting

Table 5. Estimated Detection Rate of High-Risk Lesions in Helicobacter pyloriNaïve Patients by Using the Established Model

\begin{tabular}{|lcc}
\hline $\begin{array}{l}\text { Experience of } \\
\text { endoscopy (yr) }\end{array}$ & $\begin{array}{c}\text { Observation time } \\
\text { (HP-naïv) }\end{array}$ & $\begin{array}{c}\text { Estimated detection } \\
\text { rate of HRLs } \\
\text { (HP-naïve) (\%) }\end{array}$ \\
\hline 4 & 180 & -0.04363 \\
\hline 4 & 210 & 0.04097 \\
\hline 4 & 240 & 0.12557 \\
\hline 6 & 180 & 0.039712 \\
\hline 6 & 210 & 0.124312 \\
\hline 6 & 240 & 0.208912 \\
\hline 8 & 180 & 0.123054 \\
\hline 8 & 210 & 0.207654 \\
\hline 8 & 240 & 0.292254 \\
\hline 10 & 180 & 0.206396 \\
\hline 10 & 210 & 0.290996 \\
\hline 10 & 240 & 0.375596 \\
\hline
\end{tabular}

HP, Helicobacter pylori; HRLs, high-risk lesions.
HRLs, including EGCs, on the HP-positive and HP-negative stomach. As previous reports have indicated, we found that longer observation time during screening of the stomach was a predictor for detecting HRLs. Notably, we analyzed our dataset according to the status of HP infection, defined as current infection, past infection, and HP-naïve.

EGCs generated in the atrophic mucosa after successful HP eradication therapy are difficult to detect because these EGCs usually manifest as diminutive and atypical lesions. ${ }^{13}$ Physicians recognize that HP-positive patients are at high risk for generating EGCs compared to HP-negative patients. In addition, the stomach of HP-positive patients is more difficult to screen than that of HP-negative patients due to increased mucus and active inflammation. The time required for screening the stomach is affected by such conditions. In contrast, it has been suggested that stomach with reduced inflammation post successful eradication therapy of HP tends to be screened for a shorter time compared to the HP-positive stomach. In fact, we revealed in this study that the observation time of the HP-eradicated stomach was significantly shorter than the HP-positive stomach.

Longer observation time during upper endoscopy is known to be a reliable quality indicator for detecting HRLs. ${ }^{17-19}$ As we indicated above, the observation time was affected by the status of HP infection. Nevertheless, it has yet to be determined

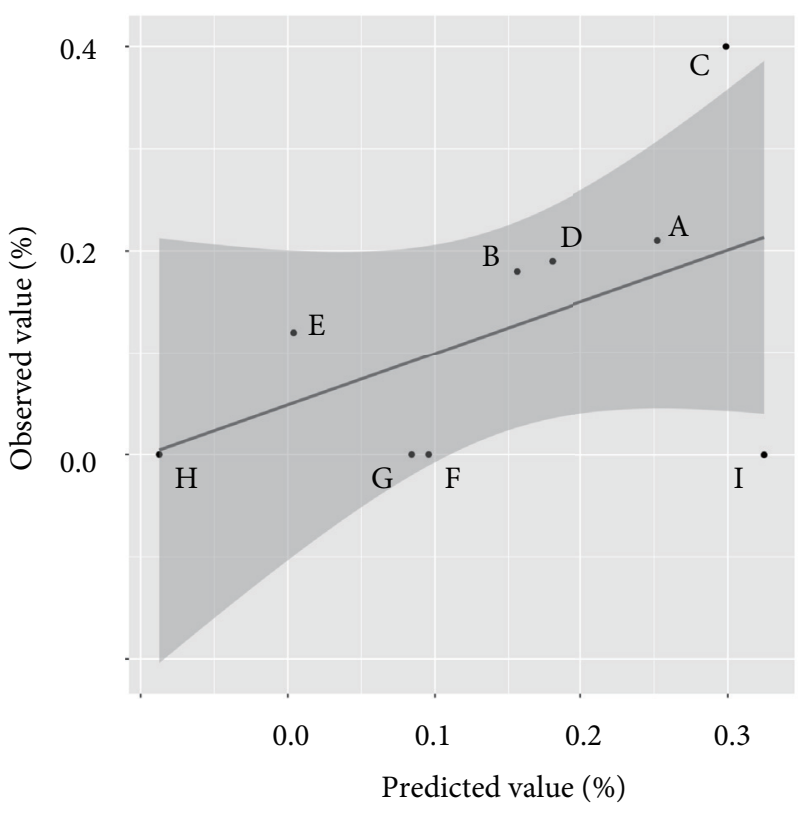

Fig. 1. Observed-predicted plot of the detection rate of Helicobacter pylori (HP)-naïve high-risk lesions (HRLs). On the basis of the equation calculated by multivariate analysis, a black line was drawn for the prediction of the physicians' detection rate of HP-naïve HRLs. The grey area indicates the $95 \%$ confidence interval. Each dot plot indicates the actual physicians' detection rate of HP-naïve HRLs. 
whether a shortened observation time lowers the detection rate of EGCs in HP-negative patients. In addition, it is not yet known whether other factors, including frequency of biopsy, are reliable quality indicators for detecting HRLs in HP-negative patients. In this study, we revealed that the observation time was a predictor for the detection of HRLs found in the HP-eradicated stomach. Thus, a shortened observation time may result in an increased risk of missing HRLs in the HP-eradicated stomach.

HP is a well-known and potent causative agent of stomach cancer. Endoscopic findings enable endoscopists to estimate the presence of HP; therefore, it is expected that detection of HP-positive HRLs might be easier than HP-negative HRLs. However, no significant predictor for detecting HP-positive HRLs was found in our study setting. In contrast, HP-naïve gastric mucosa merely generates malignant lesions, with the exception of recently reported GA-FG and other rare lesions, thus physicians have less experience of detecting HP-naïve lesions. In this study, experience of endoscopy was found to be a predictor for detecting HRLs in the HP-naive stomach, while observation time during screening the HP-naïve stomach was not. Less experienced physicians, such as trainees, should be intensively trained for detecting these rare lesions according to the training program, and screen the HP-naïve stomach carefully. Notably, the multiple regression model we established indicated that longer observation time on the HP-naïve stomach might complement less experience of the physician.

This study has several limitations. First, this study was a retrospective observation study that involved selection bias for subjects. The average age of subjects was 47.2 years old, thus this study did not cover the general population in Japan. Our cohort enclosed younger age people than the general population because most patients in our institution were healthy employees of private companies. Second, we only analyzed 37 cases of HRLs in the small cohort (a total of 13,477 cases) and only nine endoscopists were enrolled in this study; thus, the statistical power and reliability for the analyses might be weak. Third, the actual observation time of the stomach might be underestimated because we calculated the observation time retrospectively by using recorded pictures, and the time needed for the elimination of mucus, especially in the HP-positive stomach, was included in the calculation of the observation time. Fourth, judgement on the degree of atrophy of the stomach lacked inter-observer agreement in the study. Finally, it was difficult to address whether the experience of endoscopy had a continuous effect on the detection of HRL because we did not follow the performance of the same endoscopist over the years. To overcome these limitations, a large scaled-prospective study should be conducted.

In conclusion, we revealed that experience of endoscopy was a quality indicator for detecting HRLs in HP-naïve patients, whereas observation time during screening endoscopy was a quality indicator for detecting HRLs in HP-eradicated patients.

\section{Conflicts of Interest}

The authors have no financial conflicts of interest.

\section{Author Contributions}

Conceptualization: Fumiaki Ishibashi, Konomi Kobayashi, Keita Fukushima, Ryu Tanaka, Tomohiro Kawakami, Junko Kato, Kazuaki Sugihara Data curation: FI, KK, KF, RT

Formal analysis: FI, KF

Supervision: JK

Writing-original draft: FI

Writing-review\&editing: FI

\section{ORCID}

Konomi Kobayashi: https://orcid.org/0000-0001-8720-5903

Keita Fukushima: https://orcid.org/0000-0003-1258-1829

Ryu Tanaka: https://orcid.org/0000-0002-0374-0106

Tomohiro Kawakami: https://orcid.org/0000-0001-5032-6154

Junko Kato: https://orcid.org/0000-0002-6790-7657

Kazuaki Sugihara: https://orcid.org/0000-0001-8047-7578

\section{REFERENCES}

1. Nomura A, Stemmermann GN, Chyou P-H, Kato I, Perez-Perez GI, Blaser MJ. Helicobacter pylori infection and gastric carcinoma among Japanese Americans in Hawaii. N Engl J Med 1991;325:1132-1136.

2. Helicobacter and Cancer Collaborative Group. Gastric cancer and Helicobacter pylori: a combined analysis of 12 case control studies nested within prospective cohorts. Gut 2001;49:347-353.

3. Moss SF. The clinical evidence linking Helicobacter pylori to gastric cancer. Cell Mol Gastroenterol Hepatol 2017;3:183-191.

4. Uemura N, Okamoto S, Yamamoto S, et al. Helicobacter pylori infection and the development of gastric cancer. N Engl J Med 2001;345:784-789.

5. Wong BC, Lam SK, Wong WM, et al. Helicobacter pylori eradication to prevent gastric cancer in a high-risk region of China: a randomized controlled trial. JAMA 2004;291:187-194.

6. Fuccio L, Zagari RM, Eusebi LH, et al. Meta-analysis: can Helicobacter pylori eradication treatment reduce the risk for gastric cancer? Ann Intern Med 2009;151:121-128.

7. Yamamoto Y, Fujisaki J, Omae M, Hirasawa T, Igarashi M. Helicobacter pylori-negative gastric cancer: characteristics and endoscopic findings. Dig Endosc 2015;27:551-561.

8. Kobayashi M, Sato Y, Terai S. Endoscopic surveillance of gastric cancers after Helicobacter pylori eradication. World J Gastroenterol 2015;21:10553-10562.

9. Wang C, Yuan Y, Hunt RH. The association between Helicobacter pylori infection and early gastric cancer: a meta-analysis. Am J Gastroenterol 2007;102:1789-1798.

10. Matsuo T, Ito M, Takata S, Tanaka S, Yoshihara M, Chayama K. Low prevalence of Helicobacter pylori-negative gastric cancer among Japanese. Helicobacter 2011;16:415-419.

11. Ueyama H, Matsumoto K, Nagahara A, Hayashi T, Yao T, Watanabe S. Gastric adenocarcinoma of the fundic gland type (chief cell predominant type). Endoscopy 2014;46:153-157.

12. Ishibashi F, Fukushima K, Ito T, Kobayashi K, Tanaka R, Onizuka R. Influence of Helicobacter pylori infection on endoscopic findings of 
gastric adenocarcinoma of the fundic gland type. J Gastric Cancer 2019;19:225-233.

13. Saka A, Yagi K, Nimura S. Endoscopic and histological features of gastric cancers after successful Helicobacter pylori eradication therapy. Gastric Cancer 2016;19:524-530.

14. Hamashima C, Ogoshi K, Okamoto M, Shabana M, Kishimoto T, Fukao A. A community-based, case-control study evaluating mortality reduction from gastric cancer by endoscopic screening in Japan. PLoS One 2013;8:e79088.

15. Hamashima C, Ogoshi K, Narisawa R, et al. Impact of endoscopic screening on mortality reduction from gastric cancer. World J Gastroenterol 2015;21:2460-2466.

16. Hamashima C, Shabana M, Okada K, Okamoto M, Osaki Y. Mortality reduction from gastric cancer by endoscopic and radiographic screening. Cancer Sci 2015;106:1744-1749.

17. Teh JL, Tan JR, Lau LJ, et al. Longer examination time improves detection of gastric cancer during diagnostic upper gastrointestinal endoscopy. Clin Gastroenterol Hepatol 2015;13:480-487.e2.
18. Kawamura T, Wada H, Sakiyama N, et al. Examination time as a quality indicator of screening upper gastrointestinal endoscopy for asymptomatic examinees. Dig Endosc 2017;29:569-575.

19. Park JM, Huo SM, Lee HH, Lee BI, Song HJ, Choi MG. Longer observation time increases proportion of neoplasms detected by esophagogastroduodenoscopy. Gastroenterology 2017;153:460-469.el.

20. Januszewicz W, Wieszczy P, Bialek A, et al. Endoscopist biopsy rate as a quality indicator for outpatient gastroscopy: a multicenter cohort study with validation. Gastrointest Endosc 2019;89:1141-1149.

21. Kimura K, Takemoto T. An endoscopic recognition of the atrophic border and its significance in chronic gastritis. Endoscopy 1969;1:87-97.

22. $\mathrm{R}$ Core Team. $\mathrm{R}$ : a language and environment for statistical computing. $\mathrm{R}$ foundation for statistical computing [Internet]. Vienna: The R Foundation; c2018 [cited 2019 Jan 12]. Available from: https://www.R-project. org/.

23. Veitch AM, Uedo N, Yao K, East JE. Optimizing early upper gastrointestinal cancer detection at endoscopy. Nat Rev Gastroenterol Hepatol 2015;12:660-667. 